\title{
UNBOUND STATES OF THE DRIP-LINE NUCLEUS ${ }^{24} \mathrm{O}$
}

\author{
V. LAPOUX*, S. BOISSINOT, E. C. POLLACCO, F. FLAVIGNY, C. LOUCHART, \\ L. NALPAS and A. OBERTELLI \\ CEA-Saclay, DSM/IRFU SPhN, Gif-sur-Yvette, F-91191, France \\ *E-mail: vlapoux@cea.fr \\ H. OTSU, H. BABA, R.J. CHEN, N. FUKUDA, N. INABE, D. KAMEDA, \\ M. MATSUSHITA, T. MOTOBAYASHI, T. ONISHI, E.Y. NIKOLSKII, \\ M. NISHIMURA, H. SAKURAI, M. TAKECHI, S. TAKEUCHI, Y. TOGANO, \\ K. YONEDA, A. YOSHIDA and K. YOSHIDA \\ RIKEN Nishina Center, 2-1 Hirosawa, Wako, Saitama 351-0198, Japan \\ A. MATTA, Y. BLUMENFELD, S. FRANCHOO, F. HAMMACHE, PH. ROSIER and \\ E. RINDEL \\ IPN-Orsay, CNRS-IN2P3, Orsay, F-91406 France \\ P. GANGNANT, CH. HOUARNER, J.F. LIBIN and F. SAILLANT \\ GANIL, CEA/DSM - CNRS/IN2P3, Bld. H. Becquerel, Caen, F-14076, France
}

\begin{abstract}
The characteristics of the new $\mathrm{N}=16$ shell gap at the neutron drip-line can be deduced from the neutron excitations of ${ }^{24} \mathrm{O}$. An experiment was carried out to investigate the unbound excited states of ${ }^{24} \mathrm{O}$ using the proton elastic and inelastic proton scattering. It was performed in the BigRIPS line and combines the unique intensities of the RIBF ${ }^{24} \mathrm{O}$ beam with the state-of-the-art particle detector array MUST2. The method is explained.
\end{abstract}

Keywords: Unstable nuclei; shell structure; magic number; inelastic scattering.

\section{Resonances of the nuclei close to or at the drip-line}

\subsection{Motivations}

The nuclear models disagree for the predictions of the spectroscopy of nuclei far away from the valley of stability, due to the different assumptions on nucleon-nucleon interactions and few-body correlations. The studies of the structure and spectroscopy of nuclei with a high ratio of $\mathrm{N} / \mathrm{Z}$, close 
to the drip-lines, are important for our understanding of the evolution of the nuclear shell structure with the isospin. Drip-line nuclei also combine several aspects like very diffuse nuclear surfaces, halos or neutron skins, resonant states, new shell gap effects. They are weakly-bound with few or no bound excited states, and the coupling to the continuum is playing a significant role since the scattering states are much closer to the continuum states than those in stable nuclei. All these aspects are stringent tests for the microscopic structure calculations, and the drip-line nuclei appear as benchmarks for the nuclear models [1]. The existence and position of the low-lying resonant states in the light exotic nuclei would provide crucial information to constrain and to test the validity of the models.

\subsection{Resonances of the light neutron-rich ${ }^{6}$ He halo nucleus}

${ }^{6} \mathrm{He}$ has low neutron thresholds $\left(S_{n}=1.87\right.$ and $\left.S_{2 n}=0.97 \mathrm{MeV}\right)$ and no bound excited states. Under $12 \mathrm{MeV}$, only the characteristics of the first excited state, a $2^{+}$at $1.8 \mathrm{MeV}(\Gamma=113 \mathrm{keV})$ have been firmly established. Various models predict a series of $2^{+}, 1^{+}, 0^{+}$states above the $2_{1}^{+}$, but disagree on the energies of these states. We have investigated the spectroscopy of ${ }^{6} \mathrm{He}$ using the 2-neutron transfer ${ }^{8} \mathrm{He}(\mathrm{p}, \mathrm{t})^{6} \mathrm{He}$ reaction. The experiment was carried out at GANIL with the SPIRAL ${ }^{8} \mathrm{He}$ beam at $15.4 \mathrm{~A} . \mathrm{MeV}$, with no contaminant. The average intensity was $1.8 \times 10^{4} \mathrm{part} / \mathrm{s}$. The reaction target was a $50 \mu \mathrm{m}$-thick foil of polypropylene $\left(\mathrm{CH}_{2}\right)_{n}$. The beam profile and incident angle on the target were monitored event by event by two beam tracking detectors, CATS. The light charged particles were measured using 5 position-sensitive Si-strip telescopes MUST2 [2]. One MUST2 module was composed by a first stage of a $300 \mu m$ double-sided Si-strips detector (DSSD) with an active area of $10 * 10 \mathrm{~cm}^{2}$ and $128(\mathrm{x}, \mathrm{y})$ strips, each strip providing energy and time-of-flight (TOF) measurements. The DSSD is backed up by a $4 \mathrm{~cm}$-thick CsI-crystal detector, providing the remaining energy $E r$. Each strip detector has a minimum energy threshold of $0.5 \mathrm{MeV}$ with intrinsic position, angular and energy resolutions of $0.76 \mathrm{~mm}, 0.3^{\circ}$ (at $15 \mathrm{~cm}$ from the target) and $45(5) \mathrm{keV}$ (FWHM), respectively. The particles can be identified using the standard correlation techniques between the energy deposited in the Si-strip stage, $\Delta E$, and TOF, and between $\Delta E$ and $\operatorname{Er}[2]$. The excitation energy $(E x)$ spectra were calculated by the missing mass method from the measured total kinetic energy and angle of the triton detected in coincidence with forward scattered particles, either ${ }^{6} \mathrm{He}$ or ${ }^{4} \mathrm{He}$. The analysis is described in Ref. [3], two new resonances were observed: at $2.6 \pm 0.3 \mathrm{MeV}$ (width $\Gamma=1.6 \pm 0.4 \mathrm{MeV}$ ) and at $5.3 \pm 0.3 \mathrm{MeV}$ with 
$\Gamma=2 \pm 1 \mathrm{MeV}$. The angular distributions were analyzed in the framework of the coupled-reaction-channel (CRC) calculations. This method was successful to describe consistently the previous data set measured at 15.6 A.MeV: ${ }^{8} \mathrm{He}(\mathrm{p}, \mathrm{p}),(\mathrm{p}, \mathrm{d})$ and $(\mathrm{p}, \mathrm{t})$ to the ${ }^{6} \mathrm{He}$ g.s. and $2_{1}^{+}$[4]. It was found that the new states correspond to a $2^{+}$and to a $L=1$ states, respectively. The resonances were compared with the results obtained recently by various theoretical frameworks, the ab-initio calculations [5] and the models including an explicit treatment of the continuum couplings (CC) of bound and scattering states [6-8]. These CC models give calculated positions of the states rather close to the experimental values but they overestimate the width. The new states, challenging the nuclear theories, could be used as benchmarks for checking the microscopic inputs of the newly improved structure models.

\subsection{Study of the shell effect associated to $N=16$}

Experimentally the neutron drip-line is known up to the oxygen chain, with ${ }^{24} \mathrm{O}$ being the last bound isotope. In the region of the neutron-rich nuclei around ${ }^{24} \mathrm{O}$, the neutrons occupy the sd-fp shells and new effects have been found, indicated or predicted for the shell structure, in contrast with the shell occupation known for the stable nuclei. Experimentally, $\mathrm{N}=16$ has been indicated as a new possible magic number [10], theoretically interpreted as an enhancement of the neutron shell gap between the $2 s_{1 / 2}$ and $1 d_{3 / 2}$ subshells, with respect to the sd shell in the stable nuclei, this effect being driven by the tensor proton-neutron force [11]. To explore the properties of this new shell effect, the low-lying spectroscopy of ${ }^{24} \mathrm{O}$ was studied: at GANIL, no gamma-ray associated to ${ }^{24} \mathrm{O}$ was found [12], showing that the excited states are unbound. At MSU, via invariant mass method from ${ }^{24} \mathrm{O}$ fragments and neutrons, possible states were indicated [13] around 4.5 and $5.3 \mathrm{MeV}$, above the neutron threshold $S_{n}$ at $4.19 \mathrm{MeV}$ [9], but not clearly identified. An alternative technique to study the neutron excitation is to measure the proton elastic and inelastic scattering, and to use the particle spectroscopy.

\section{Spectroscopy of ${ }^{24} \mathrm{O}$ using the (p,p') reaction}

From the detection of the recoil proton the (p,p') kinematics can be reconstructed and the $E x$ spectrum can be obtained via the missing mass method. Our ${ }^{24} \mathrm{O}(\mathrm{p}, \mathrm{p}$ ') measurement became feasible with the advent of the RI Beam Factory operated by RIKEN Nishina Center and CNS, Uni- 
versity of Tokyo. The experiment (RIBF57) was performed in 2010 using the MUST2 detector array and the secondary beams produced by the fragmentation on a Be target of the ${ }^{48} \mathrm{Ca}$ beam at $345 \mathrm{~A} . \mathrm{MeV}$. The beams were separated using the BigRIPS line [14]. With the exceptional rates of ${ }^{48} \mathrm{Ca}$, (60 up to 180 particle $\mathrm{nA}$ ), ${ }^{24} \mathrm{O}$ was produced at $263 \mathrm{~A} . \mathrm{MeV}$ with unique intensities, $1100 / \mathrm{s}$ on average (2000, maximum).

\subsection{Experimental set-up and kinematics}
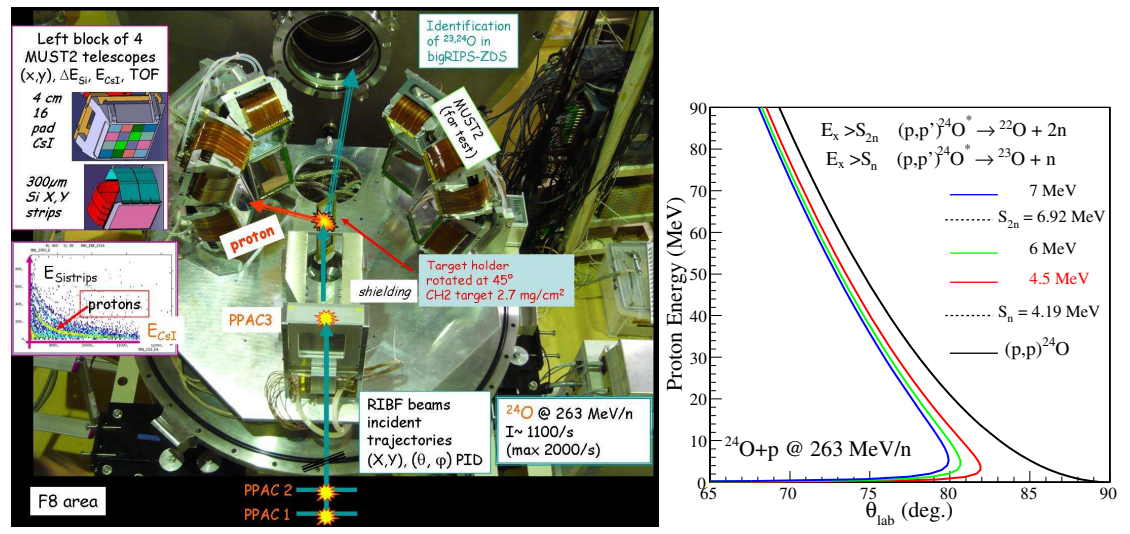

Fig. 1. Top view of the set-up inside the reaction chamber; plot of the ${ }^{24} \mathrm{O}$ (p,p') kinematics calculated for three hypothetical states, located at $4.5 \mathrm{MeV}, 6$ and $7 \mathrm{MeV}$.

The reaction chamber was mounted in the $\mathrm{F} 8$ area with 8 MUST2 detectors, assembled in two blocks. The $2.7 \mathrm{mg} / \mathrm{cm}^{2}$-thick $\left(\mathrm{CH}_{2}\right)$ reaction target was rotated at $45 \mathrm{deg}$. towards the left block. The left telescopes were located at $23 \mathrm{~cm}$ downstream of the target, to cover lab. angles between (65-90) deg. The experimental set-up located in the F8 area is presented in Fig. 1 with the angular and energy domain covered by the left block. The measured angular domain for the elastic cross sections is between $5-30_{c . m}^{\circ}$. The (p,p') kinematics were obtained by the correlations of the proton energies with the scattering angle deduced from the proton trajectories measured in MUST2 and from the incident trajectories reconstructed using 3 PPACs located upstream of the target. 


\subsection{Particle identification}

The incident particles were identified by $\mathrm{B} \rho$-ETOF- $\Delta E$ technique in the (F3-F7) stages, and the ejectiles in the ZD [15] spectrometer. The rate of ${ }^{24} \mathrm{O}$ represented $4 \%$ of the total beams, including the light nuclei with $\mathrm{A} / \mathrm{Z}=3\left(\mathrm{t},{ }^{6} \mathrm{He},{ }^{9} \mathrm{Li}\right)$. Amongst the heavy ion beams $(\mathrm{Z}>3)$, it represented $22 \%$ of the total; contaminants were ${ }^{20,21,22} \mathrm{~N}(\simeq 30 \%),{ }^{22,23} \mathrm{O}(\simeq 20 \%),{ }^{25} \mathrm{~F}$ $(\simeq 12 \%)$ and ${ }^{26} \mathrm{~F}(\simeq 5 \%)$.

\section{The analysis of the $E x$ spectra}
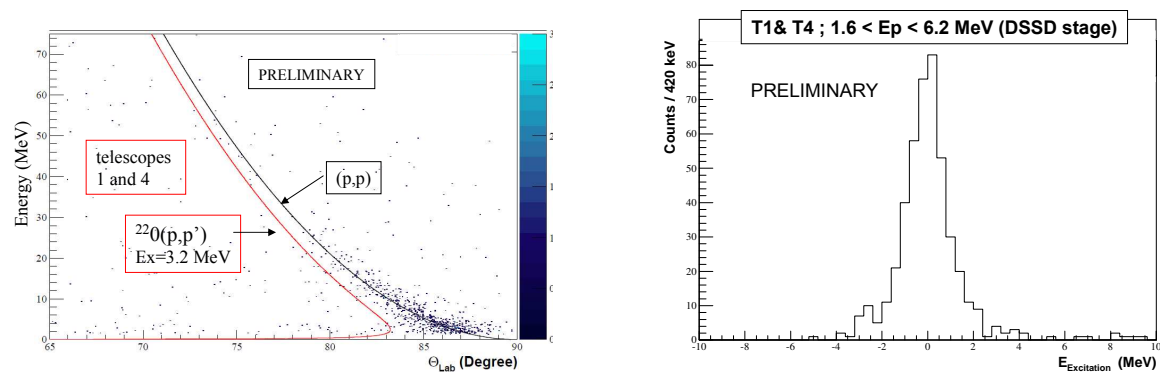

Fig. 2. The kinematics and $E x$ spectra for ${ }^{22} \mathrm{O}$, showing the known $2_{1}^{+}$at $3.2 \mathrm{MeV}$.

To check the kinematical reconstruction of the reactions, the ${ }^{22} \mathrm{O}(\mathrm{p}, \mathrm{p}$ ') at $262.5 \mathrm{~A}$.MeV were measured as a reference. The kinematics and the preliminary Ex spectra are shown in Fig. 2, the energy straggling effects in the target are taken into account.

The next steps are the extraction of the excitation spectra for ${ }^{22} \mathrm{O}$ and for its contaminants. The angular acceptance and detector efficiencies will be evaluated and compared with the estimates done using a Monte-Carlo simulation program. The elastic (p,p) angular distributions will be extracted and compared to model calculations. We will check the consistency of the data obtained for ${ }^{22} \mathrm{O}$ with the previous measurements done at GANIL at $46 \mathrm{MeV} / \mathrm{n}$ [16]. The analysis is also in progress for ${ }^{24} \mathrm{O}\left(\mathrm{p}, \mathrm{p}^{\prime}\right)$. The data was collected with different settings of the magnetic rigidity tuned to center either the ${ }^{23} \mathrm{O}$ fragment or the ${ }^{22} \mathrm{O}$ at the focal plane of the spectrometer. For the event selection and the reconstruction of the ${ }^{24} \mathrm{O} E x$ spectra we will take into account the correlations with the outgoing nucleus produced by the decay of the unbound states of ${ }^{24} \mathrm{O}$, either ${ }^{23} \mathrm{O}\left(E x\right.$ above $\left.S_{n}\right)$ or 
the ${ }^{22} \mathrm{O}$ isotope (above $S_{2 n}=7.5 \mathrm{MeV}$ ).

\section{Perspectives}

The results will be compared with the recent microscopic calculations, including the interplay between cluster, single-particle and continuumcoupling effects $[7,17,18]$. From the characteristics of the excited states, we expect to deduce the properties of the $\mathrm{N}=16$ shell gap in ${ }^{24} \mathrm{O}$.

\section{Acknowledgments}

Special thanks are due to the accelerator team of the RIBF machine for delivering the ${ }^{48} \mathrm{Ca}$ at maximum intensities. RIKEN Nishina Center is gratefully acknowledged for supporting the stay of the French team during the campaign.

\section{References}

1. T. Otsuka et al., Phys. Rev. Lett. 105, 032501 (2010).

2. E. Pollacco et al., The MUST2 collaboration, Eur. Phys. J. A 25, 287 (2005).

3. X. Mougeot, V. Lapoux et al., submitted to Phys.Rev. Lett., June 2011.

4. N. Keeley et al., Phys. Lett. B 646, 222 (2007).

5. S. C. Pieper, R. B. Wiringa, and J. Carlson, Phys. Rev. C 70, 054325 (2004).

6. N. Michel, et al., Phys. Rev. C 67, 054311 (2003).

7. A. Volya and V. Zelevinsky, Phys. Rev. Lett. 94, 052501 (2005).

8. G. Hagen, M. Hjorth-Jensen, J.S. Vaagen, Phys. Rev. C 71, 044314 (2005).

9. Evaluation of mass excess 2011, Priv. Co. (Apr. 2011) G. Audi and W. Meng.

10. A. Ozawa et al., Phys. Rev. Lett. 84, 24 (2000).

11. T. Otsuka et al., Phys. Rev. Lett. 91, 179202 (2003).

12. S. Stanoiu et al., Phys. Rev. C 69, 034312 (2004).

13. C.R. Hoffman et al., Phys.Lett. 672, 17 (2009).

14. T. Kubo et al., NIM. Phys. Res. B 204, 97 (2003).

15. T. Ohnishi et al., J. Phys. Soc. Jpn. 77, 083201 (2008).

16. E. Becheva et al.,MUST collaboration, Phys. Rev. Lett. 96, 012501 (2006).

17. K. Tsukiyama, M. Hjorth-Jensen, G. Hagen, Phys. Rev. C 80, 051301 (2009).

18. K. Tsukiyama, T. Otsuka and R. Fujimoto, arXiv:1001.0729 (2010). 